\title{
Preparation and Properties of Sweat-gland Type High Temperature Self-lubricating Metal Ceramic Preform
}

\author{
HAN Tian Tian ${ }^{1, a}$, WANG Yan Jun ${ }^{1, b^{*}}$, WANG Shou Ren ${ }^{1, c}$ and LI Shu Long ${ }^{1, d}$ \\ ${ }^{1}$ School of Mechanical Engineering,University of Ji'nan, Ji'nan,250022,China \\ a1015773343@qq.com, b me_wangyj@ujn.edu.cn, \\ csherman0158@tom.com, ${ }^{\mathrm{d}} 1101701864 @ q q . c o m$,
}

\begin{abstract}
Keywords: high temperature self-lubricating; cermet sinter; porosity; mechanical properties Abstract. A compound pore forming agent which can be decomposed constantly in sintering process was designed and prepared. A new cermet sinter was developed by adding the compound pore forming agent into the TiC/M2 matrix material. The influences of content of $\mathrm{TiC}$, sintering temperature and pore forming agent content on the porosity and mechanical properties of preforms were analysed.The results show that: the sintering bodies with different properties can be prepared by changing the matrix content, sintering parameters and pore forming agent content.The preform with interpenetrating network structure can be prepared by adding $20 \% \mathrm{TiC}, 6 \%$ pore forming agent, and sintering at $1220^{\circ} \mathrm{C}$ for 60 minutes.
\end{abstract}

\section{Introduction}

With the development of modern science and technology, the friction, wear and lubrication of the materials at high temperature are drawing more and more attention, which promotes the development of high temperature self-lubricating materials[1].The traditional method of preparing high temperature self-lubricating composite is sintering by adding solid lubricant to metallic or ceramic matrix. However, in the process of high-temperature sintering, the solid lubricant is easy to be oxidated, which will cause loss to its lubricating properties; at the same time, the uneven distribution of solid lubricant and the damage to continuity of matrix will significantly reduce the strength, toughness and wear resistance of composite. Based on the structure of sweat-gland, the high temperature self-lubrication micro-porous materials are prepared by infiltrating solid lubricants into micro-porous metal ceramic preforms, which can effectively realize self-lubricating under some special conditions. The material has become an area of "intense research" [2-3].

Xin Laicheng[4] et al. prepared Ni-Co-Al materials with different Co content by powder metallurgy.They noted that the moderate amount of Co can improve the hardness, compressive strength and the tribological properties of the alloy, but the excessive Co (Co mass fraction of 30\%) made a worse tribological properties of the alloy.So the research of balancing the strength and the lubrication of the self-lubricanting material is an important part of designing these materials.Author designed the based materials and the pore forming agent, then prepared high temperature self-lubricating preform and studied the effection of main compositions and sintering conditions on porosity and mechanical properties, which will be helpful to prepare high temperature self-lubricating preform with certain porosity and mechanical properties.

\section{Design of Main Ingredients}

Design Principle. Metal ceramic sintering body with micro-porous structure has four components: hard phase, tough phase, adhesive and pore forming agent.For the metal ceramic composites, the matching of ceramic and metal has great influence on interfacial stress and the material properties. So it is necessary to promise the matching of the elastic modulus and the coefficient of thermal expansion between each phase. Meanwhile, making it sure that strong chemical reactions cat not take place between each phase. The matching degree of the elastic modulus will determine the degree of the matrix crack can be prevented.The lager differernts of elastic modulus will reduce the material strength and toughness, and even make the product cracking. The differernts of thermal expansion coefficient of each phase can cause thermal stress, which will cause material micro crack and a loss of 
material strength and toughness. The difference of the expansion coefficient between the two phases should be less than $5 \times 10^{-6}{ }^{\circ} \mathrm{C}$ in case the product destroyed[5]. At the same time, strong chemical reactions that can cause a certain phase to be lost or badly eroded are not allowed to occur between any two phases.In addition, the wettability should also be considered. The wetting angle between metal and ceramic should be no more than $90^{\circ}$, or by adding certain agent to make the wetting angle eligible.

Design of Based Materials. The lubrication and mechanical properties of the materials can be improved by adding appropriate amount of element. Li Jianliang et al. prepared the nickel matrix composites by powder metallurgy method. They noted that the friction and wear properties of the composites were improved by adding 6\%-12\% graphite[6]. Author designed the composition of micro-porous metal ceramic sintered body: TiC ceramic powder (hard and tough phase); M2 alloy powder (bonded phase); $\mathrm{Cu}$ (increasing the wettability of each phases); $\mathrm{Co}$ (improving the plasticity of the metal ceramic material); $\mathrm{Cu}_{3} \mathrm{P}$ (reducing the sintering temperature).

Design of Compound Pore Forming Agent. Single pore forming agent whose decomposing temperature range is limited[7].So it is difficult to form a high porosity with a single pore forming agent. Pore forming agent should be decomposed constantly in the process of sintering in order to form preform whose structure is similar to human sweat-gland structure( the shape of pore is regular, the internal pores are interconnected to the network). The sintered body which is prepared by adding this pore forming agent has a higher open porosity that makes it easy to infiltrate and effuse the solid lubricant.The shape and distribution of pore can be controlled by controlling the amount, shape ,distribution of pore forming agent[8-9].Author designed the composition of the compound pore forming agent: $\mathrm{CaCO}_{3}, \mathrm{TiH}_{2}$ and $\mathrm{Al}_{2} \mathrm{O}_{3}$. TiH $\mathrm{Ti}_{2}$ produces $\mathrm{H}_{2}[10]$, then $\mathrm{CaCO}_{3}$ produces $\mathrm{CO}_{2}$, that is, gas will be produced almost in the whole process of sintering. The matrix do not infiltrate the $\mathrm{Al}_{2} \mathrm{O}_{3}$ whose distribution is dispersive. So in the process of sintering, pore can be prevented from shrinkage and closure by using this pore forming agent.

\section{Experimental Procedure}

Analysis of Thermal Property. The thermal property of compound pore forming agent was studied by differential thermal analyzer. The sample was placed in a ceramic crucible, and the ceramic crucible was placed in the thermal analyzer.The heating rate was $10^{\circ} \mathrm{C} / \mathrm{min}$, and the temperature range was from room temperature to $1200^{\circ} \mathrm{C}$.

Preparation of Preform . The mixture was homogenized in a ball mill for 3 hours. The proportion of mixture and steel ball was 1:4. The powder milled was compressed into sample whose size was $\varphi 12 \times 20$ by $500 \mathrm{MPa}$ forming pressure. The sintered body was prepared by ZT-70-20Y type vertical vacuum hot pressing furnace at $1200^{\circ} \mathrm{C}, 1220^{\circ} \mathrm{C}, 1240^{\circ} \mathrm{C}$ for 60 minutes. The heating rate is $5^{\circ} \mathrm{C} / \mathrm{min}$. Keeping warm at compound pore forming agent disappeared temperature for 20 minutes.

Performance Testing. Porosity of preforms were analyzed using hydrostatic method[11].The crushing strength of preforms were tested by YE-600 type hydraulic testing machine.

\section{Results and Discussion}

TG-DSC Analysis . Fig. 1 is the TG-DSC curves of pore forming agent. It shows: with the increase of temperature, the water and zinc stearate volatilize gradually, which produces $0.88 \%$ weight loss at $336.2^{\circ} \mathrm{C}$. From $336.2^{\circ} \mathrm{C}$ to $519.4^{\circ} \mathrm{C}$, the weight loss of pore forming agent is seldom.From $519.4^{\circ} \mathrm{C}$ to $729.9^{\circ} \mathrm{C}$, the weight of pore forming agent is much reduced, which indicates compound pore forming agent is mainly decomposed in this stage. Compound pore forming agent can be decomposed constantly in a broad temperature range (from $336.2^{\circ} \mathrm{C}$ to $1144.6^{\circ} \mathrm{C}$ ), which is helpful to ensure the continuity of the pores. 


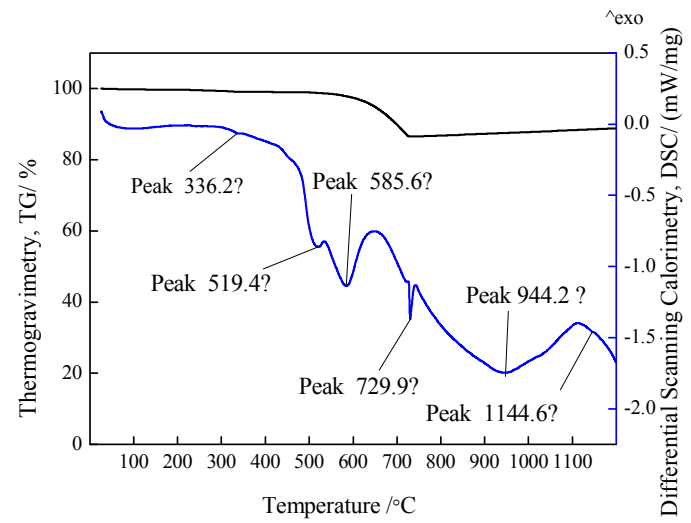

Fig. 1 TG-DSC curves of compound pore-forming agent

Effect of Matrix Content. Fig.2 is the influence of $\mathrm{TiC}$ content on the properties of ceramic sinters. The results in Fig. 2(a) show: with the increase of TiC content, the crushing strength and the density of the material decrease; with the increase of pore forming agent, the strength and the density of the material decrease. The results in Fig. 2(b) show: with the increase of TiC content, the open porosity of the material increases; with the increase of pore forming agent, the open porosity of the material increases. Take the effect of $\mathrm{TiC}$ content on the porosity and mechanical properties of the sintered body into account, adding $20 \% \mathrm{TiC}$ to prepare preform.

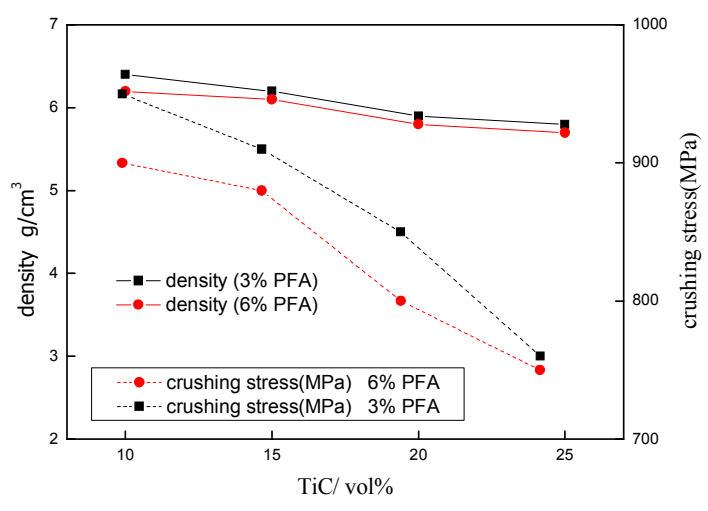

(a)content of TiC-density and crushing strength

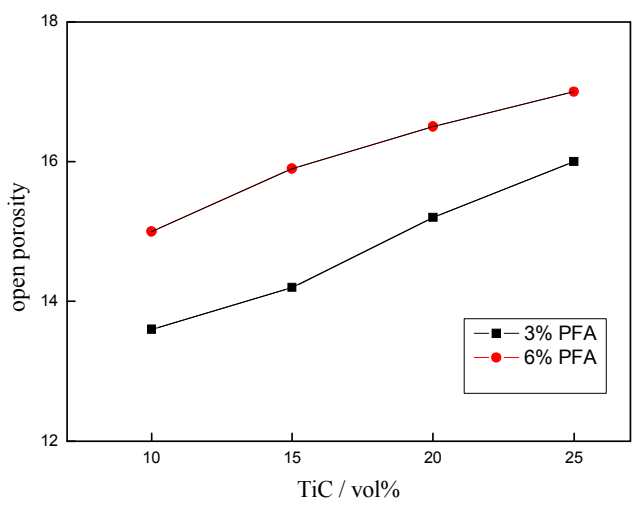

(b)content of TiC-open porosity

Fig.2 The influence of content of $\mathrm{TiC}$ on the properties of ceramic sinters

Effect of Sintering Temperature. The sintered bodies were perpared by adding $6 \%$ pore forming agent. Fig.3(a) shows: with the rise of sintering temperature, the porosity of the preform decreases; Fig.3(b) shows: with the rise of sintering temperature, the crushing strength increases. After weighting the request for pore structure and obdurability, it is good to sinter at $1220^{\circ} \mathrm{C}$.

Effect of Pore Forming Agent Content. The sintered bodies were perpared at $1220^{\circ} \mathrm{C}$. Fig. 4 is the curves of influence of pore forming agent content on porosity and crushing strength. Fig.4(a) shows: with the increase of pore forming agent,the total porosity of the preform increases; Fig.4(b) shows: with the increase of pore forming agent,the crushing strength of the preform decreases.After weighting the request for pore structure and obdurability, it can help to add 4-8\% pore forming agent. 


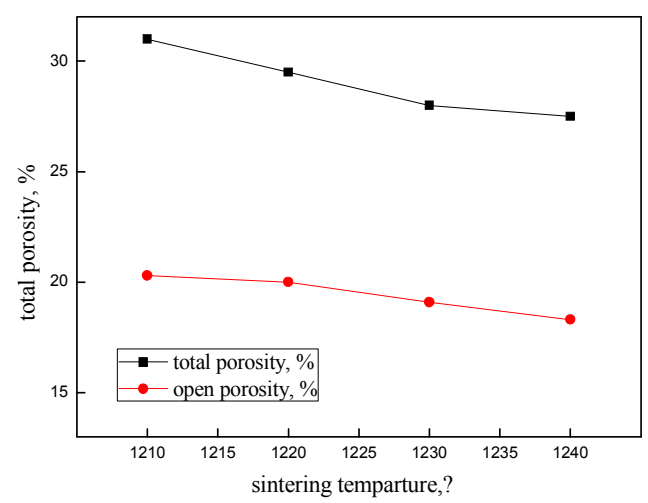

(a) sintering temperature-porosity

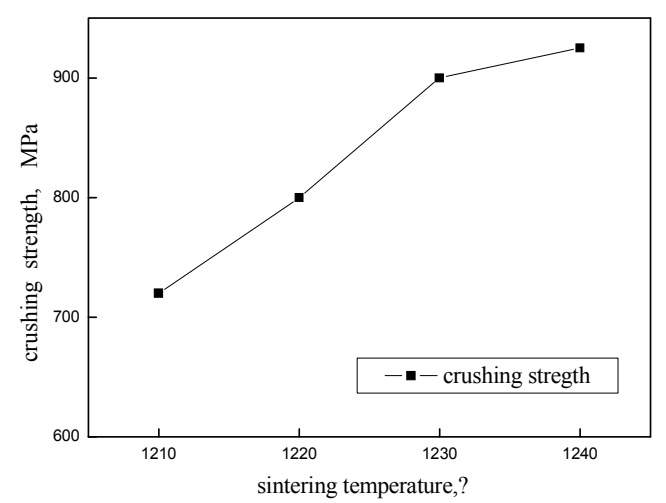

(b) sintering temperature-crushing strength

Fig. 3 The influence of sintering temperature on porosity and crushing strength

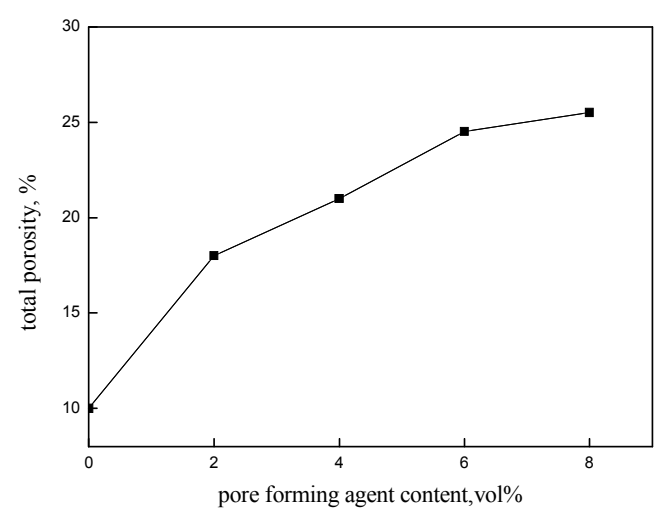

(a) pore forming agent content-total porosity

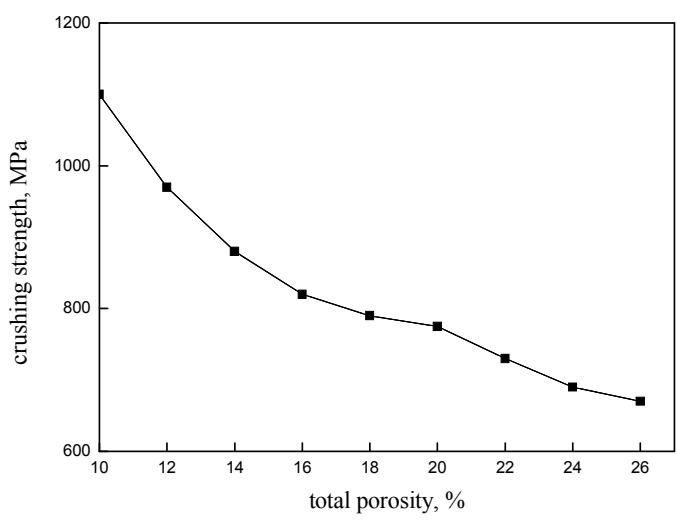

(b) total porosity -crushing strength

Fig.4 The influence of pore forming agent content on porosity and crushing strength

Microstructure. TiC/M2 based metal ceramic micro-pore perform was sintered by adding $20 \%$ $\mathrm{TiC}, 6 \%$ pore forming agent at $1220^{\circ} \mathrm{C}$ for 60 minutes. The microstructure of it was analyzed using scanning electron microscope (SEM). Fig. 5 shows that: the internal pores of the sintered bodies are mutually communicated, and the longitudinal pores are tubular, which makes it easy to infiltrate solid lubricant.

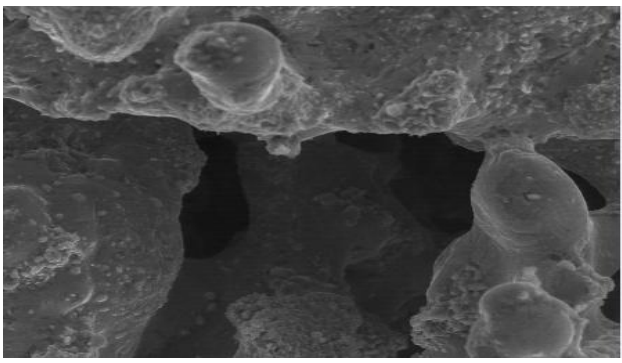

(a) lateral drawing;

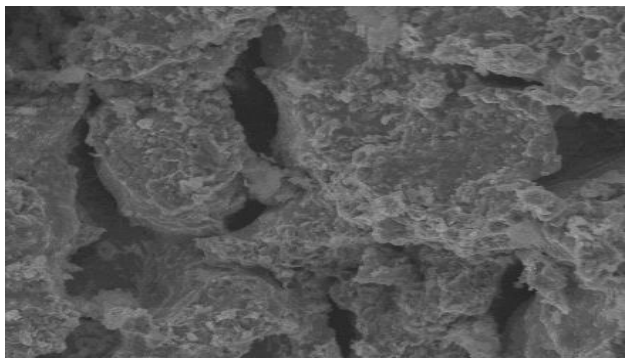

(b) longitudinal drawing

Fig.5 Pores SEM micrographs of TiC/M2 based metal ceramic micro-pore perform

\section{Summary}

The sintering bodies with different properties can be prepared by changing the matrix content, sintering parameters and pore forming agent content. After weighting the request for pore structure and obdurability, a technic of preparation was made: sintering at $1220^{\circ} \mathrm{C}$ for 60 minutes by adding $20 \% \mathrm{TiC}, 6 \%$ pore forming agent. In this way, the preform with interpenetrating network structure which makes it easy to infiltrate solid lubricant can be prepared . 


\section{Acknowledgement}

The authors would like to thank the National Natural Science Foundation of P.R. China for the financial support (ID: 51275208).

\section{References}

[1] AiFang Wang, Dingjun Zhang, Youzhi Wu, Effects of adding $\mathrm{MoS}_{2}$ and graphite on tribological properties of Ni-Cr based self-lubricating composites, Chinese Journal of Material Research. 24(2010)464-470.

[2] Ping Lu, Zuomin Liu, Study on the precipitation ability of lubricant based on its cellular structure and constituents,Lubrication Engineering. 35(2010)37-41.

[3] X. F. Shi , B. L. Sitharaman , Fabrication of porous ultra-short single-walled carbon nanotube nanocomposite scaffolds for bone tissue engineering, Biomaterials. 28(2007) 4078-4090.

[4] Chenglai Xin, Wenzhen Wang, Effect of Co content on the tribological properties of a Ni-Co-Al Superalloy, Journal of materials research. 28(2014) 490-496.

[5] Yanjun Wang, Zuomin Liu, Design of diffusing self-lubricating and wear-resisting ceramic sinter, Materials for Mechanical Engineering. 29(2005)12-15.

[6] Jianliang Li, Dangsheng Xiong, Tribological behavior of graphite-containing nickel-based composite as function of temperature, load and counterface, Wear. 266(2009)360-367.

[7] Yong Chen, Preparation and characterization of porous materials, Press of University of Science and Technology of China, Hefei,2010.

[8] Zhang H L, Li J F, Micro-structure and electrical properties of porous PZT ceramics derived from different pore-forming agents, Acta Materialia. 55(2007) 171-181.

[9]Minhua Luo, The practical technology of porous ceramics, China Building Materials Press, Beijing, 2006.

[10]Guangming Huang, Study on preparation of Ti powder by dehydrogenation,Tanium Industry Progress.27(2010)6-9.

[11]Yingzi $\mathrm{Gu}$, Zhaohui Chen, Uncertainty evaluation of density measurements by hydrostatic weighing, Measurement and Equipment. 6(2006)8-11. 